\title{
Using a Wide Area Receiver Network to Support GBAS Ionospheric Monitoring
}

\author{
Maria Caamano, Daniel Gerbeth, Michael Felux, Mihaela-Simona Circiu, \\ German Aerospace Center (DLR)
}

\section{BIOGRAPHIES}

Maria Caamano received a Master's degree in Telecommunications Engineering from the University of Oviedo, Spain, in March 2015. During Master studies, she specialized in the field of signal theory and communications. The same year she joined the German Aerospace Center where she is working on the impact of ionospheric irregularities in the single-frequency single-constellation GBAS.

Daniel Gerbeth received a Bachelor and Master's degree in Electrical Engineering and Information Technology from Karlsruhe Institute of Technology, Germany in 2014. During Master studies he specialized in aerospace technology and navigation. After working in the field of sensor fusion and navigation aiding at Fraunhofer IOSB he joined German Aerospace Center (DLR) in May 2015 and is involved in the research on GBAS since then.

Michael Felux received a diploma in Technical Mathematics from the Technische Universität München in 2009. The same year he joined the German Aerospace Center where he was working on the development of the GBAS GAST C testbed at the research airport in Braunschweig and its upgrade to GAST D. He was involved in flight testing and ground validation of the station and the approach procedures. Since 2015 he is coordinating DLR's research on GBAS-based navigation.

Mihaela-Simona Circiu studied Computer Engineering at Technical University Gheorghe Asachi, Romania. She obtained a 2nd level Specialized Master in Navigation and Related Application from Politecnico di Torino, Italy in 2012. Since 2013 she has been working as a research associate at the Institute of Communications and Navigation at the German Aerospace Center. Her main focus is multi-frequency multi-constellation Ground Based Augmentation System.

\footnotetext{
ABSTRACT

Ionospheric anomalies, like ionospheric gradients, might produce a difference between the ionospheric error experienced by the Ground Based Augmentation System (GBAS) reference station and the aircraft on approach. This ionospheric delay difference could lead to position errors if undetected.

For that reason, the GBAS Approach Service Types (GAST) C and D provide solutions against this threat, but the methods employed still face challenges. In the case of GAST C, the GBAS integrity parameters are inflated in order to exclude potentially usable satellite geometries that could produce unacceptably large position errors if affected by a worst case gradient. The GAST D concept contains a significant amount of monitors to cover different regions of the ionospheric threat space, which includes a combination of gradient sizes, directions and speeds. Both methodologies have a negative impact on the availability of the system, especially when trying to implement GAST C and D in regions with severe ionospheric behavior, i.e. equatorial regions, because of the exclusion of too many satellite geometries in the case of GAST C and a high rate of false alarms in GAST D.

This paper proposes a real-time ionospheric monitoring approach that supports the already existing GAST C and D solutions without changing the overall integrity concept. The proposed concept is based on a multi-frequency multi-constellation receiver network external to the GBAS installation that provides real-time ionospheric information to the GBAS stations. A GAST C reference station could use this information to reduce the maximum gradient to consider in the inflation of the integrity parameters while a GAST D station could benefit from prior probabilities of appearance relaxing the monitoring thresholds and thus provoking less false alarms.

The monitoring approach is first explained and then evaluated with simulated data. This data is constructed based on real measurements of noise and multipath taken with both Choke-ring and Multipath Limiting Antennas (MLAs), and models for the nominal ionospheric decorrelation.
} 
Simulation results show that a considerable reduction of the threat space that the remaining monitors in GAST C and D have to consider seems feasible. Furthermore, the main effects of the presence of high levels of noise and multipath in the measurements used are discussed.

\section{INTRODUCTION}

The Ground Based Augmentation System (GBAS) is a local-area augmentation of the Global Navigation Satellite Systems (GNSSs). Its main purpose is to 'augment' or enhance the GNSS performances in terms of integrity, continuity, accuracy and availability. A GBAS reference station broadcasts differential corrections along with integrity parameters. The differential corrections enable the aircraft approaching the airport to correct the navigation signals from the satellites and thus improve the accuracy of its position. The integrity parameters enable the airborne system to calculate bounds of the residual position errors and ensure the safety of the operation.

The currently commercially available GBAS Approach Service Type (GAST) C ground stations support operations down to CAT I minima [1]. Standards for GAST D intended to support CAT II/III operations are completed and stations could be operational by 2020. Both GAST C and GAST D systems are based on a single-frequency (L1) single-constellation (GPS) architecture.

Single-frequency single-constellation differential corrections provided by the GBAS ground station enable the airborne system to correct most of the GPS ranging errors, especially the ones caused by the experienced ionospheric delay. Nevertheless, nominal residual errors still remain and are overbounded by the so-called protection levels (PLs). The protection levels are calculated by the avionics using error models and the integrity parameters received from the ground station. Then, they are compared to the alert limits (ALs), the maximum allowable bounds, to determine safety of the operation for each user. If the PLs exceed the ALs, GBAS is unavailable. The component of the PLs that describes and overbounds the remaining residual errors due to the nominal ionospheric decorrelation between the reference station and the user is called the $\sigma_{v i g},[2]$, [3]. $\sigma_{v i g}$ is an integrity parameter broadcast by the ground station that ensures the safety of the operation only as long as the state of the ionosphere is nominal. Thus, it does not protect users against abnormal ionospheric activity, like large ionospheric gradients acting between the ground station and the airplane on approach. In this case, there is a significant difference between the ionospheric error experienced by the GBAS reference station and the aircraft. This may lead to not differentially-corrected and improperly bounded position errors if undetected.

Protection of airborne users against this kind of threat has already been tackled in GAST C and D. A GAST C ground station verifies by simulation that each satellite geometry potentially usable at the aircraft (PLs $<$ ALs) is safe in the presence of the ionosphere-anomaly threat applicable in the region in a process called geometry screening [4]. In case a simulated satellite geometry is not safe, the ground station inflates the integrity parameters so that the PLs exceed the ALs when an arriving aircraft aims to use this satellite geometry, making GBAS unavailable. The main problem is that in GAST C, this algorithm is performed by applying a threat model based on the worst-ever-experienced ionospheric gradient in the relevant region, e.g. $140 \mathrm{~mm} / \mathrm{km} \mathrm{in}$ the case of Germany [3] or $425 \mathrm{~mm} / \mathrm{km}$ in the CONUS region [5]. This methodology is very conservative because it assumes that the worst-case ionospheric gradient is always present in the ionosphere, while this is very unlikely. This means that satellite geometries are excluded even in days with nominal ionosphere (without a gradient) leading to a loss of availability. Moreover, this procedure cannot protect users against a larger gradient that might occur at some point in time in the future.

The GAST D concept follows a different approach when protecting against harmful ionospheric gradients. The ground manufacturer has to ensure that the maximum differential range error due to an ionospheric gradient at every Landing Threshold Point (LTP) supporting GAST D operations is not greater than 2.75 meters [6]. For that purpose, GAST D does not assume always the presence of a worst-case gradient as done in GAST C, but contains a significant amount of sensitive monitors on the ground as well as in the airborne system [7].

Combining all the monitors together, the GAST D GBAS should prevent the ionospheric differential range error to exceed the mentioned limit by detecting all the possible ionospheric gradients inside the threat space with a probability of missed detection $\left(P_{m d}\right)$ of $10^{-9}$. The threat space is the combination of all possible gradient sizes, velocities and directions that can occur in a certain region up to a maximum, which depends again on the worst gradient ever seen. The monitoring thresholds were evaluated utilizing the mid-latitude ionospheric threat model, which goes up to $500 \mathrm{~mm} / \mathrm{km}$ [8]. While trying to adapt the GAST D concept to other regions, the monitors could suffer from a high rate of false alarms (as in equatorial regions) impacting the GBAS availability or even be unnecessary (as the Ionospheric Gradient Monitor in Germany).

In short, the methods employed in GAST C and D are able to ensure the required level of safety, but can result in undesirable unavailability of the system, especially in equatorial regions with highly active ionospheric conditions. Here, the ionospheric conditions are completely different from those of the mid-latitudes, where the ionosphere is nominally 'calm' and rarely presents large ionospheric gradients harmful for GBAS.

Many studies have been carried out in this direction, evaluating the possibility of implementing GBAS in equatorial regions without losing significantly its availability. In [9] and [10] the assessment of the Brazilian GBAS ionospheric spatial gradient was carried out including gradients up to $850.7 \mathrm{~mm} / \mathrm{km}$ produced by Equatorial Plasma Bubbles (EPBs), which are ionospheric anomalies, smaller in size but often steeper than the ionospheric gradients observed in the CONUS region. In [11], the authors develop the concept of the GAST C geometry screening for Brazil including as well the EPBs. They calculate the availability of 


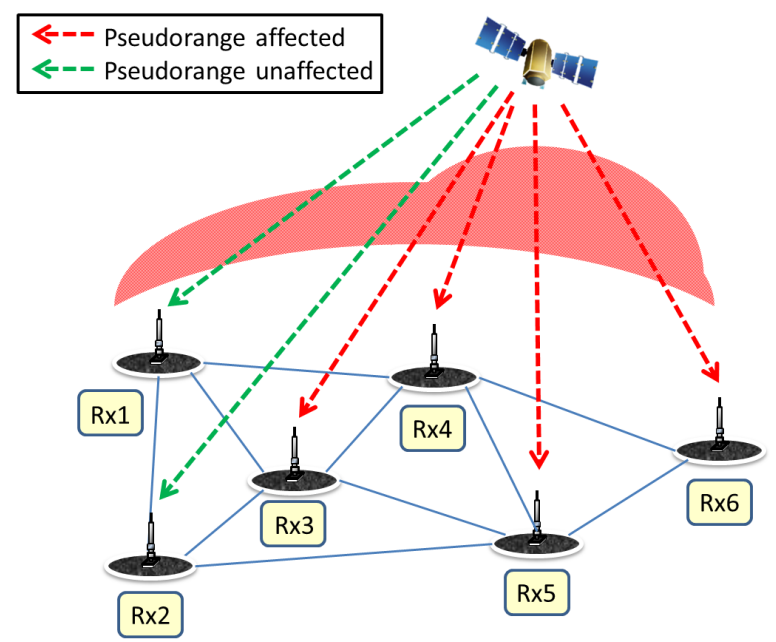

Figure 1: Simplified scheme of the network.

GBAS to be $99.3 \%$ during the daytime and $58.3 \%$ in the nighttime when using the Brazilian threat model. Their results show the difficulty to meet CAT I availability requirements while using the geometry screening and propose a Morte Carlo method that improves the availability results but is still insufficient. Furthermore, these methods can still not protect against future larger gradients because they rely on the ionospheric threat model, which is based on historical data.

In the case of GAST D, the service provider may consider that the ionospheric behaviour in the region he is in charge of is not adequately characterized by the threat model. In this situation, he is allowed to introduce additional internal or external monitoring to the GBAS (section 7.5.6.1.7. 'Ionospheric Anomaly Threat Models Used for GAST D validation' of the International Civil Aviation Organization (ICAO) Standards And Recommended Practices (SARPs) [6]).

In this work, we propose a real-time ionospheric monitoring approach that supports the already existing GAST C and D solutions by reducing their conservatism without changing the overall integrity concept. The result of our monitoring defines the maximum gradient to be used in the GBAS with a resultant reduction of the threat space in both GAST C and D.

\section{METHODS}

\section{A. Dual frequency monitoring concept}

As mentioned previously, the availability of GBAS is determined mainly through the achieved protection levels and the rate of false alarms. The first ones are increased especially in GAST C while assuming a worst-case gradient that is rarely present. The second is problematic in GAST D while applying the same monitoring thresholds to regions in which the ionosphere is not represented with the mid-latitudes ionospheric threat model [8].

For that reason, the goal of this work is to introduce some flexibility in the current GAST C and D approaches and thus increase their availability by introducing external information in GBAS. This external information would come from a widespread network of receivers located outside the GBAS installation. In Figure 1, we show a simplified scheme of the proposed network with only six receivers and one satellite, but the concept can be expanded for $n$ receivers and $m$ satellites from different constellations.

The functionality of the network is described in the following:

1. The network monitors the ionosphere in real-time. For each epoch, ionospheric delays are estimated at each Ionospheric Pierce Point (IPP). The Ionospheric Pierce Points (IPPs) are the intersections of the line of sight satellite-receiver with the ionosphere, modelled as a thin shell located at $350 \mathrm{~km}$ from the Earth's surface [12].

2. Ionospheric gradients between all the ionospheric delay estimations are calculated. Here, all the possible connections between receivers are taken into account, i.e. $R x_{i}$ and $R x_{j}$ where $i \in[1,6]$ and $j \in[1,6]$ in the example of Figure 1 . If both IPPs are unaffected by the gradient, we measure the nominal spatial decorrelation of the ionosphere.

3. The estimated ionospheric gradients are shared in the network.

4. Each GBAS station is then responsible of using the network information to support the already existing equipment in covering the GBAS approaches of each airport. The minimum detectable gradient is calculated for every GBAS station taking into 
account the multipath and noise conditions, the location of the GBAS station and the network information for this location. Finally, the integrity parameters are adjusted according to the detector performance. Therefore, the overall integrity concept would remain unchanged.

Let us remark that this monitoring approach would not replace the inflation in GAST C or the monitors in GAST D, but would reduce the threat space that the current techniques have to cover. The introduction of this concept implies: i) in GAST C, the substitution of the always considered worst-case gradient used in the geometry screening with the minimum detectable gradient achieved with the proposed network and ii) in GAST D, the relaxation of the current monitoring thresholds plus added detectability of ionospheric gradients not covered by the mid-latitudes ionospheric threat space. As an outcome, we expect less stringent monitoring requirements to be necessary with the consequent increase in the availability of current GAST C and D.

\section{B. Description of the estimation approach}

Our network consists of a set of multi-frequency multi-constellation receivers distributed over a region, that will detect the gradients and provide integrity to the single-frequency single-constellation users in real time. The multi-frequency capability is used to estimate the ionospheric delays, whereas the multi-constellation may bring a benefit while detecting the gradients for the single-constellation users as we dispose of more measurements of the ionospheric delay. In this paper, we use two constellations: GPS and Galileo.

In the following, we describe how the network works in real time. For each epoch:

1. All the receivers acquire the pseudorange measurements from the dual-frequency satellites, which are then smoothed with a Hatch Filter. The smoothed pseudorange measurements are expressed in Equation 1 [13]:

$$
\widehat{\rho}_{i}^{j}=r^{j}+c\left(\delta_{t}-\delta_{t}^{j}\right)+\widehat{T}^{j}+\widehat{I}_{i}^{j}+\widehat{M P}_{i}^{j}+\widehat{\xi}_{\rho, i}^{j}
$$

where $r^{j}$ is the geometric range from user to the satellite $j, c$ is the speed of light, $\delta_{t}$ is the receiver clock bias, $\delta_{t}^{j}$ is the satellite clock bias, $\widehat{T}^{j}$ is the smoothed tropospheric delay, $\widehat{I}_{i}^{j}$ is the smoothed ionospheric delay for frequency $i$ and $\widehat{M P}_{i}^{j}$ and $\widehat{\xi}_{\rho, i}^{j}$ are the code multipath and thermal noise on frequency $i$. The frequency $i$ is L1/E1 (1575.42 MHz) and L5/E5a (1176.45 $\mathrm{MHz}$ ) for both GPS and Galileo.

In the next paragraphs, we consider the superscripts as satellites and the subscripts as frequencies.

2. The algorithm computes the dual frequency ionospheric slant delay (Equation 2) [13]:

$$
\widehat{I}_{f_{1}, \text { slant }}^{j}=\frac{f_{2}^{2}}{f_{1}^{2}-f_{2}^{2}}\left(\widehat{\rho}_{f_{2}}^{j}-\widehat{\rho}_{f_{1}}^{j}\right)
$$

where $f_{2}$ is $L 5$ for the GPS and $E 5 a$ for Galileo and $f_{1}$ is $L 1$ for the GPS and $E 1$ for Galileo.

If we substitute in Equation 2, the expression of the smoothed pseudoranges from Equation 1, we obtain:

$$
\widehat{I}_{f_{1}, \text { slant }}^{j}=\frac{f_{2}^{2}}{f_{1}^{2}-f_{2}^{2}}\left(\widehat{\Delta I}_{f_{2}, f_{1}}^{j}+\widehat{\Delta M P}_{f_{2}, f_{1}}^{j}+\widehat{\Delta \xi}_{f_{2}, f_{1}}^{j}+\widehat{\eta}_{f_{2}, f_{1}}^{j}\right)
$$

where $\widehat{\Delta I}_{f_{2}, f_{1}}^{j}, \widehat{\Delta M P} f_{f_{2}, f_{1}}^{j}$ and $\widehat{\Delta \xi}_{f_{2}, f_{1}}^{j}$ are the differences of the ionospheric delay, code multipath and code thermal noise between the smoothed pseudoranges in $f_{1}$ and $f_{2}$. Moreover, an additional term $\widehat{\eta}_{f_{2}, f_{1}}^{j}$ appears here, which represents the interfrequency biases introduced on the code by the receivers and the satellites.

The problem of using the slant delay for estimating an ionospheric gradient is that not all the satellites are comparable because of the path that the pseudoranges have to follow through the ionosphere, which is longer when the satellites are in low elevations (the minimum is just in the zenit). Because of the elevation dependency, only the satellites with parallel line of sights would be comparable, and this fact reduces the number of IPPs available to detect ionospheric gradients.

3. For that reason, the slant delay $\left(\widehat{I}_{f_{1}, \text { slant }}^{j}\right)$ is translated into vertical delay by applying the obliquity factor (OF) expressed in Equation 4 [13] like in Equation 5.

$$
O F^{j}=\left[1-\left(\left(\frac{R_{E}}{R_{E}+h_{I}}\right) \cos \left(\theta^{j}\right)\right)^{2}\right]^{\frac{-1}{2}}
$$


Here, $R_{E}$ is the radius of the Earth, $h_{I}$ is the height of the thin shell layer commonly set at $350 \mathrm{~km}$ above the Earth's surface and $\theta^{j}$ is the elevation of satellite $j$ in degrees.

$$
\widehat{I}_{f_{1}, \text { vert }}^{j}=\frac{\widehat{I}_{f_{1}, \text { slant }}^{j}}{O F^{j}}
$$

This way, all the measurements are comparable, but other issues may arise: the OF is only valid as long as the ionosphere is nominal. This means that we could commit an error in case of using it in the situation of having a gradient as well. In this work, we neglect this issue as we consider the calculation of this error and introduction of its value in our algorithm as part of the future work.

4. Once we have in each epoch all the vertical ionospheric delays, we compute the gradients between all the possible IPPs by applying the following formula:

$$
\text { Vertical_ionospheric_gradient }{ }^{j, k}=\frac{\widehat{I}_{f_{1}, v e r t}^{j}-\widehat{I}_{f_{1}, v e r t}^{k}}{d^{j, k}}
$$

where the Vertical_ionospheric_gradient is commonly expressed in $\mathrm{mm} / \mathrm{km}, \widehat{I}_{f_{1}, \text { vert }}^{j}$ and $\widehat{I}_{f_{1}, \text { vert }}^{k}$ are the ionospheric vertical delays in milimeters in L1/E1 and $d^{j, k}$ is the distance between the IPPs. Let us remark that the gradient slopes in this case are calculated in the vertical domain and not in slant as in the threat model [5].

As seen in Equation 3 and mentioned previously, in a real case, the estimation of the slant ionospheric delay does not contain only information about ionosphere, but combined multipath, thermal noise and interfrequency biases from two pseudoranges as well. These measurements depend strongly on the antenna and receiver characteristics as well as their location on ground. As a consequence, it may happen that the value of the ionospheric delay is estimated incorrectly due to the level of noise and multipath present in the measurements. These errors affect particularly when using them to estimate a gradient (Equation 6) as they gain importance when the IPPs of the measurements are very close together and thus we divide by a small distance. Putting a hard limit on the IPP distances that we use for our calculations could be a solution, but as we are especially looking for close by distances to better detect the gradients, it is necessary to characterize the local noise and multipath affecting the measurements and determine their effect in the value of the ionospheric delay. Besides, even without any harmful irregularities present in the ionosphere, we can always find the nominal ionospheric decorrelation in our estimation of the gradient.

For that reason, in this work we study the ionospheric gradients that could be detected utilizing a network with the characteristics described in Section A. In other words, we compute the minimum gradient that could be detected with different characteristics of noise and multipath. The tuning of the integrity parameters or the evaluation of the concept with actual gradients will be part of the future work. Moreover, further studies will have to be done to account for the interfrequency biases mentioned in Equation 3 .

\section{Simulation}

At this point, step 4 of Section A., we need to compute the minimum detectable gradient for the current epoch by using the Vertical_ionospheric_gradients computed with Equation 6.

This value is derived considering the uncertainty of our approach, $\sigma_{\text {monitoring }}$, which includes both the uncertainty of the noise and multipath of the measurements as well as the uncertainty of the nominal vertical ionospheric delay.

In this work, we calculate the minimum detectable gradient with simulated data based on real measurements. We construct the $\sigma_{\text {monitoring }}$ by introducing known levels of noise and multipath acquired from real measurements to the nominal vertical ionospheric delay.

The real levels of code noise and multipath are simulated considering two different types of antennas: Choke-ring and Multipath Limiting antennas (MLAs). The first ones lead to more noise and multipath, but are much cheaper than the seconds ones, which we use as a reference for good quality measurements. For representing the nominal ionospheric conditions, we take samples from the Klobuchar model [12] and the $\sigma_{v i g}$ value calculated for Germany [3].

Once we have both the uncertainties of noise and multipath and the ionospheric nominal decorrelation, we need to combine them to compute the minimum detectable gradient that our network can achieve. This process is described in the following.

\section{Noise and multipath}

In the GBAS context, the local system characteristics, multipath and noise, are represented in the so-called $\sigma_{g n d}$ values. These values for $\sigma_{g n d}$ are derived from at least 24 hours of measurements from the ground station and are based on a 100-second smoothing time constant. In this work, we use measurements from two different types of antennas: Leica AR-25 Choke ring antennas situated in DLR's GBAS test bed in Braunschweig (northern Germany) and MLAs [14]. 
As we want to study the effects of the combined multipath and noise from two frequencies, we need to compute the combination of two $\sigma_{g n d}$ values, one from $f_{1}$ and the other one from $f_{2}$ as represented in Equation 7 [15]:

$$
\sigma_{g n d_{f_{1}, f_{2}}}=\sqrt{\left(\frac{f_{1}^{2}}{f_{1}^{2}-f_{2}^{2}}\right) \sigma_{g n d, f_{1}}^{2}+\left(\frac{f_{2}^{2}}{f_{1}^{2}-f_{2}^{2}}\right) \sigma_{g n d, f_{2}}^{2}}
$$

where $f_{1}$ is the GPS L1 frequency $1575.42 \mathrm{MHz}, f_{2}$ is the GPS L5 frequency $1176.45 \mathrm{MHz}, \sigma_{g n d, f_{1}}$ are the $\sigma_{g n d}$ values for the frequency L1 and $\sigma_{g n d, f_{2}}$ are the $\sigma_{g n d}$ values for the frequency L5. All $\sigma_{g n d}$ values were computed by sorting data into elevation bins with bin sizes of $1^{\circ}$ up to a satellite elevation of $30^{\circ}, 2^{\circ}$ between $30^{\circ}$ and $50^{\circ}$, and $5^{\circ}$ for all higher elevations.

Let us remark that we have taken into account $\sigma_{\text {gnd }}$ values computed only with GPS satellites, as we consider Galileo with equal or better performance in terms of multipath and noise [16].

As we have two satellites involved in the calculation of the vertical ionospheric gradient, in this value the combination of the multipath and noise from both satellites is present. We calculate the standard deviation of the combined noise and multipath from both satellites, $\sigma_{\text {multipath_noise_comb }}$, in Equation 8.

$$
\sigma_{\text {multipath_noise_comb }}\left(\theta^{j}, \theta^{k}\right)=\sqrt{\sigma_{g n d_{f_{1}, f_{2}}}^{2}\left(\theta^{j}\right)+\sigma_{g n d_{f_{1}, f_{2}}}^{2}\left(\theta^{k}\right)}
$$

where $\theta^{j}$ is the elevation of one of the satellites and $\theta^{k}$ is the elevation of the other satellite involved in the calculation of the vertical ionospheric gradient. The only variables present in this calculation are the elevations of both satellites due to the fact that the noise and multipath of the measurements are not dependent on the separation between their IPPs, but they are on the elevation of the satellites.

\section{Nominal spatial decorrelation of the ionosphere}

As previously stated, the ionosphere changes nominally with space and time. This phenomenom is called ionosphere spatial and temporal decorrelation. The spatial decorrelation produces an expected difference in the value of the vertical delay because the ionosphere is being sampled in two or more IPPs that are separated. In other words, the larger it is the distance between two IPPs more likely it is that the value of the ionospheric vertical delay is different in each of them, and the closer together, the less difference there is in these values.

This means that, besides the noise and multipath, we need to consider as well the ionospheric decorrelation in our calculations in order to have a better estimation of the detection capability.

As mentioned in Section I., the $\sigma_{v i g}$ value describes the nominal spatial decorrelation of the ionosphere per country/region. However, a GBAS ground station cannot distinguish typical 'quiet' ionospheric conditions from 'active' (but not stormy) conditions in real time [2]. For that reason, data from 'active' but not harmful ionospheric conditions was taken into account in the $\sigma_{v i g}$ calculation. Therefore, it makes sense to use it as a conservative overbound of the nominal state of the ionosphere in our simulations. We use as well the Klobuchar model [12] to determine a less conservative nominal decorrelation of the ionosphere.

\section{Klobuchar model}

The goal of the Klobuchar model [12] is to be able to correct at least the 50 percent of the ionospheric time-delay in single-frequency single-constellation global positioning systems. It includes the main features of the complex behavior of the ionosphere in an algorithm that requires a minimum of coefficients and user computational time.

The Klobuchar model provides the ionospheric time-delay correction for GPS given geodetic latitude, geodetic longitude, elevation angle and azimuth angle to the particular GPS satellite and $\alpha_{n}$ and $\beta_{n}$ coefficients, which are transmitted as part of the satellite message [12]. The ionospheric vertical time-delay can then be translated into spatial delay by multiplying by the speed of light. Once the vertical ionospheric delay is estimated in each IPP with the Klobuchar model, Equation 6 can be applied to calculate all the vertical ionospheric gradients between the IPPs. However, in order to obtain a representative value for the nominal decorrelation using the Klobuchar model, we build a Gaussian distribution by using all the vertical ionospheric gradients previously calculated during one day. Below, we explain the process to compute the Klobuchar distribution used in this paper.

For every 300 seconds in a day, we compute the IPPs corresponding to GPS and Galileo satellites by using information from their nominal almanacs and 30 receivers uniformly randomly distributed over Germany. The date of the data used is: 16-08-2016. Then, we calculate the ionospheric vertical delay for that IPPs using the Klobuchar model [12]. After that, we compute the vertical ionospheric gradients in milimeters per kilometer between IPPs and store them as well as the distance between the corresponding IPPs. All the data is sorted into distance bins and mean and $\sigma$ values are computed inside each bin. Each of the $\sigma$ values per distance bin is overbounded. The result is our $\sigma_{k l o b u c h a r}$, which depends only in the distance between the IPPs. 


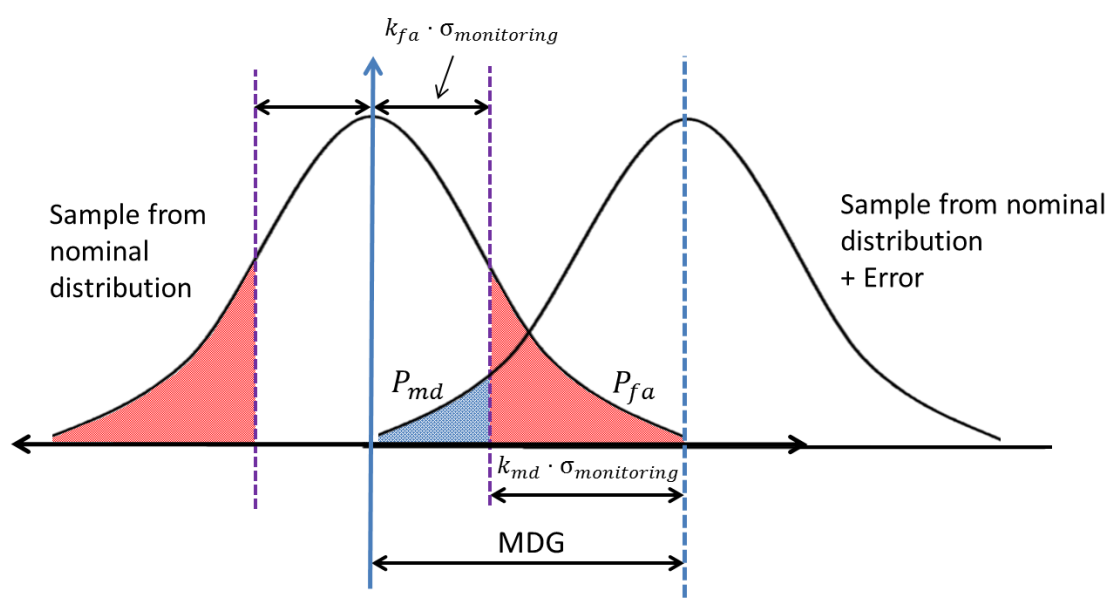

Figure 2: Minimum Detectable Gradient.

\section{Minimum Detectable Gradient calculation}

Considering both the noise and multipath component, $\sigma_{\text {multipath_noise_comb }}$ computed with Equation 8, and the ionospheric vertical decorrelation component, $\sigma_{\text {decorr }}$ that is either $\sigma_{\text {vig }}$ or $\sigma_{\text {klobuchar }}$, we can calculate our $\sigma_{\text {monitoring }}$ as in Equation 9.

$$
\sigma_{\text {monitoring }}\left(\theta^{j}, \theta^{k}, d^{j, k}\right)=\sqrt{\sigma_{\text {decorr }}^{2}\left(d^{j, k}\right)+\sigma_{\text {multipath_noise_comb }}^{2}\left(\theta^{j}, \theta^{k}\right)}
$$

Here, $\theta^{j}$ is the elevation of satellite $j, \theta^{k}$ is the elevation of satellite $k$ and $d^{j, k}$ is the distance between the IPPs corresponding to satellites $j$ and $k$.

A sample from the nominal distribution is represented in Figure 2 as Gaussian with zero mean and a standard deviation of $\sigma_{\text {monitoring }}$ as we include the nominal ionospheric decorrelation as part of its uncertainty. Given acceptable false alarm and missed detection probabilities, $P_{f a}$ and $P_{m d}$, a minimum detectable gradient (MDG) can then be defined as:

$$
M D G=\left(k_{f a}+k_{m d}\right) \cdot \sigma_{\text {monitoring }}\left(\theta^{j}, \theta^{k}, d^{j, k}\right)
$$

where $k_{f a}$ is the false alarm multiplier computed from the inverse of the standard normal cumulative distribution (Equation 11) and $k_{m d}$ is the missed detection multiplier (Equation 12).

$$
\begin{aligned}
& k_{f a}=Q^{-1}\left(\frac{P_{f a}}{2}\right) \\
& k_{m d}=Q^{-1}\left(\frac{P_{m d}}{2}\right)
\end{aligned}
$$

\section{RESULTS AND DISCUSSION}

\section{A. Noise and multipath}

Figure 3 shows that the largest combined noise and multipath, $\sigma_{\text {multipath_noise_comb }}$, for the Choke-ring antenna coincides with the situation of having both satellites at $5^{\circ}$ elevation. The $\sigma_{\text {multipath_noise_comb }}$ in this case is equal to 0.75 meters due to the fact that the noise and multipath get larger when the satellites are in low elevations. On the other hand, noise and multipath have less importance when the satellites are located on high elevations, e.g. when $\theta^{j}$ and $\theta^{k}$ are both $85^{\circ}$, which results in a value of $\sigma_{\text {multipath_noise_comb }}$ of 0.25 meters.

In the case of using a MLA (Figure 4), the combined noise and multipath values are all below 0.1 meters, making an important difference with the case of the Choke-ring antenna.

Moreover, we can observe a difference in the shape of both Figures 3 and 4 . This difference is due to the noise and multipath limitation capability of the MLA, which decreases the dependence of these values on the elevation. This way, the noise and multipath values represented in Figure 4 are very similar to each other regardless of the elevation of the satellites. 


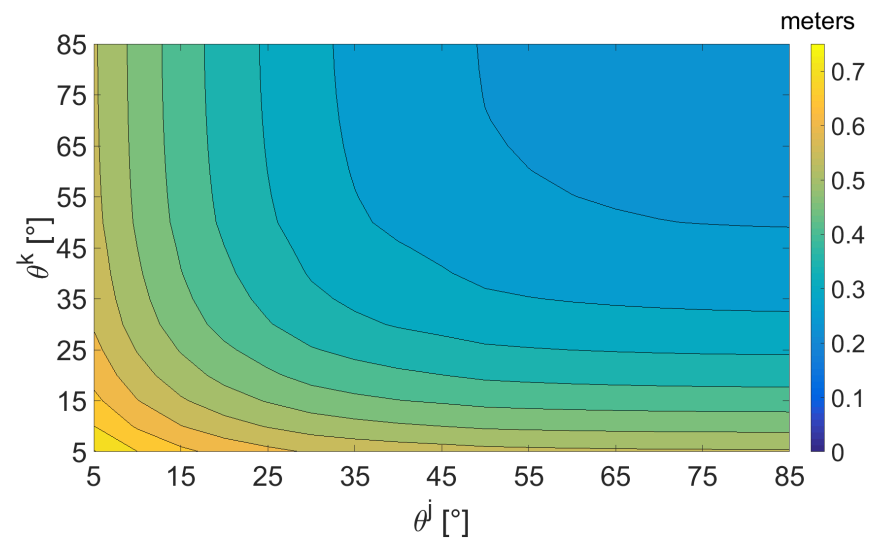

Figure 3: $\sigma_{\text {multipath_noise_comb }}$ for the Choke-ring antenna.

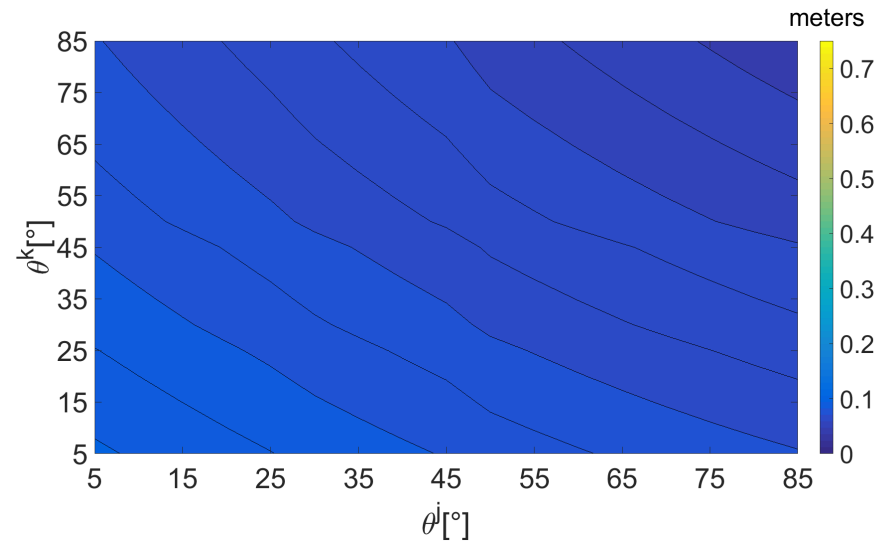

Figure 4: $\sigma_{\text {multipath_noise_comb }}$ for the MLA.

\section{B. Nominal spatial decorrelation of the ionosphere}

In this subsection, we compare the nominal ionospheric decorrelation explained in Section C., Germany's $\sigma_{v i g}$ and $\sigma_{k l o b u c h a r}$, with the level of noise and multipath presented in the previous subsection. In this case, we eliminate the elevation dependence by calculating the $\sigma_{\text {multipath_noise_comb }}$ of the actual satellites, GPS and Galileo, in their elevations during one day and then sorting the calculated values into IPP distance bins.

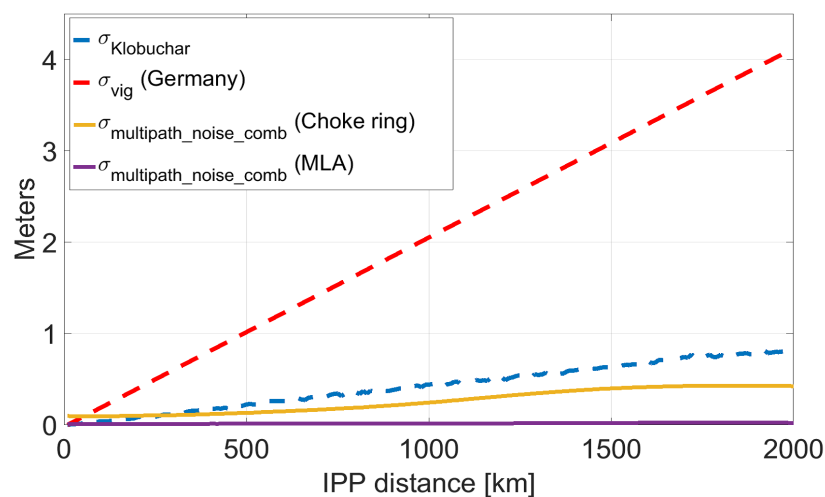

Figure 5: Comparison between $\sigma_{\text {multipath_noise_comb }}$ (Choke-ring and MLA) and the nominal ionospheric decorrelation $\left(\sigma_{\text {klobuchar }}\right.$ and $\left.\sigma_{v i g}\right)$.

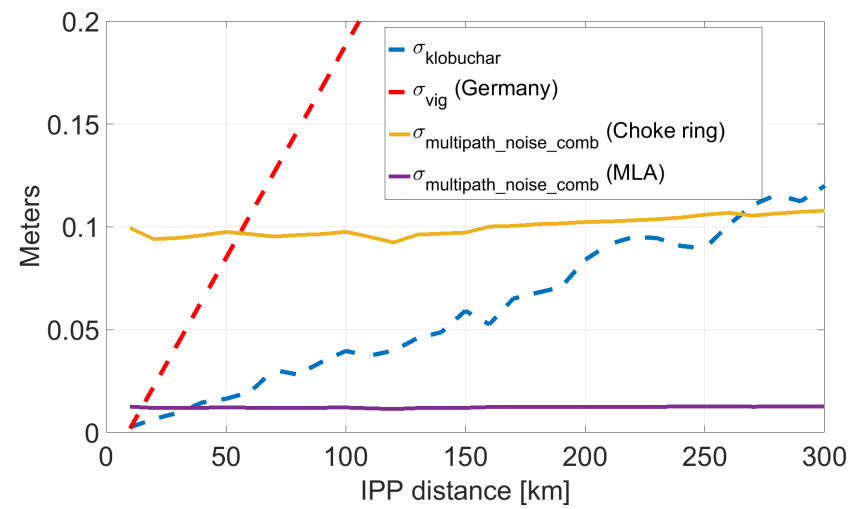

Figure 6: Zoom of the comparison between

$\sigma_{\text {multipath_noise_comb }}$ (Choke-ring and MLA) and the nominal ionospheric decorrelation $\left(\sigma_{\text {klobuchar }}\right.$ and $\left.\sigma_{\text {vig }}\right)$.

Figures 5 and 6 show that until an IPP separation distance of $270 \mathrm{~km}$, the nominal decorrelation of the ionosphere calculated with the Klobuchar model does not exceed the noise and multipath that a Choke-ring antenna would measure. This means that for distances below this value, we could detect a gradient when there are only noise and multipath. In the case of the MLA, the nominal ionosphere exceeds the noise and multipath at a much shorter IPP separation distance, $37.5 \mathrm{~km}$. This is already a small distance between IPPs considering the study of the $\sigma_{v i g}$ computation in which the authors pointed out the lack of data for IPP separation distances below $50 \mathrm{~km}$ [2]. Our approach would increase the information about the ionosphere considerably, as we could use close-by IPPs to compute the gradients. In the case of the conservative bound for the nominal decorrelation, $\sigma_{v i g}$, the value decreases to $10 \mathrm{~km}$.

However, in this subsection we are not taking into account yet the GBAS integrity requirements. In the next subsection, we include the probabilities of false alarm and missed detection, $P_{f a}$ and $P_{m d}$, mentioned in Section II. C. in order to introduce sufficient integrity into the concept and therefore meet the GBAS requirements.

\section{Minimum Detectable Gradient calculation}

Considering the $\sigma_{\text {multipathnoise_comb }}$ and the $\sigma_{\text {decorr }}, \sigma_{\text {vig }}$ and $\sigma_{\text {klobuchar }}$, represented in Figures 5 and 6, we calculate $\sigma_{\text {monitoring }}$ expressed in Equation 9. Therefore, the minimum detectable gradient (Figures 7, 8 and Equation 10) is computed by fixing both the $P_{m d}$ to $10^{-9}$ and $P_{f a}$ to $10^{-7}$. 


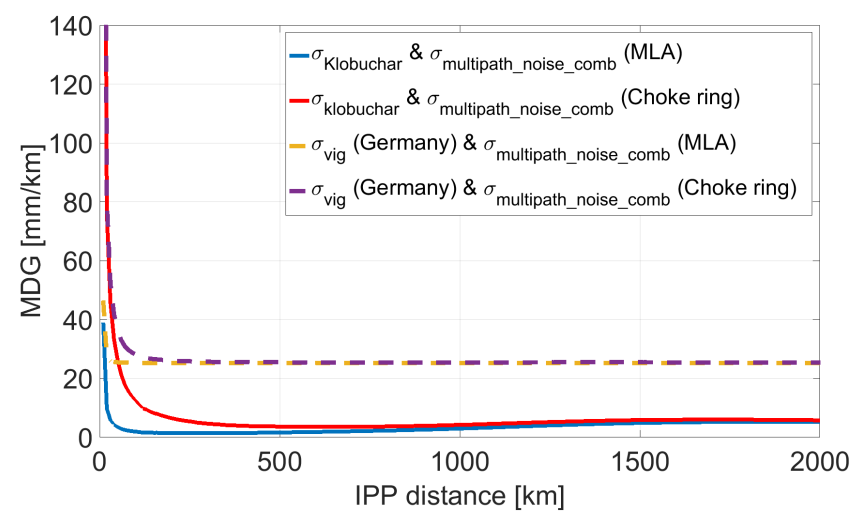

Figure 7: Minimum detectable gradient for all the combinations of antennas (Choke-ring and MLA) and nominal ionospheric decorrelations $\left(\sigma_{\text {klobuchar }}\right.$ and $\left.\sigma_{\text {vig }}\right)$.

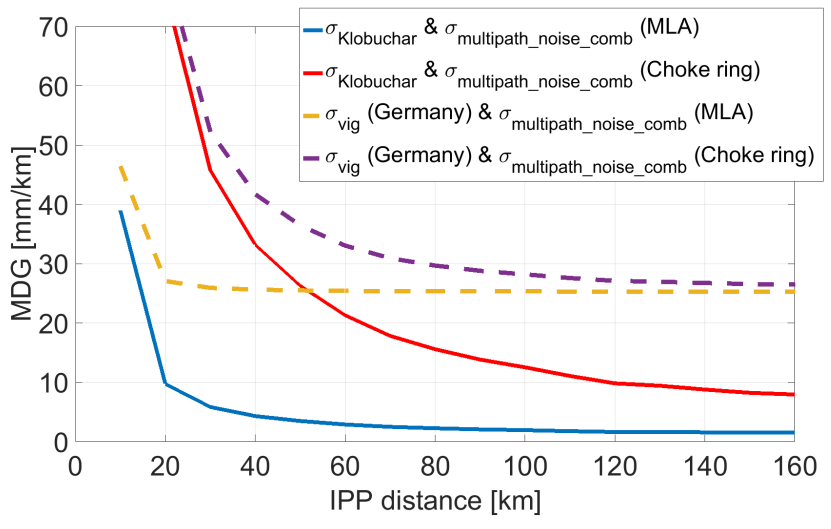

Figure 8: Zoom of the minimum detectable gradient for all the combinations of antennas (Choke-ring and MLA) and nominal ionospheric decorrelations $\left(\sigma_{\text {klobuchar }}\right.$ and $\left.\sigma_{\text {vig }}\right)$.

Results show the minimum slope of the ionospheric gradient that our network would be able to detect taking into account different conditions of noise, multipath and nominal decorrelation. This result only applies if the direction of movement of the gradient is parallel to the line connecting the IPPs used for the gradient estimation. In case the direction is different, we could only estimate the projection of the actual gradient into the parallel line already mentioned.

Regarding this information, a considerable reduction of the threat space that has to be considered while performing ionospheric monitoring in GBAS seems feasible.

In the most conservative scenario, using Choke-ring antennas and $\sigma_{v i g}$ (dashed-violet line in Figures 7 and 8), we can detect gradients above $45 \mathrm{~mm} / \mathrm{km}$ with IPP separation distances over $38 \mathrm{~km}$. This result implies three main facts: i) we can not distinguish between an actual gradient and high amounts of noise and multipath when using IPP separation distances below $38 \mathrm{~km}$ in this worst-case scenario, ii) we could miss an actual gradient which is below $45 \mathrm{~mm} / \mathrm{km}$ and iii) we could underestimate the value of an ionospheric gradient when it is very narrow and steep because the sampling of the ionosphere is still not enough compared to the gradient size.

Nevertheless, we are able to lower considerably the value currently used in the German threat model, $140 \mathrm{~mm} / \mathrm{km}$, by approximately 3 times for baselines over $38 \mathrm{~km}$ and directions of the gradient parallel to the IPPs location.

In a more realistic scenario, Choke-ring antennas and the Klobuchar model (red line in Figures 7 and 8 ) we can detect gradients over $45 \mathrm{~mm} / \mathrm{km}$ for IPP separation distances over $30 \mathrm{~km}$. Here, we are able to use data coming from IPPs separated $30 \mathrm{~km}$ for the same gradient value. There is a difference with the previous result as we are considering a less conservative nominal ionosphere, but we are still not able to use the most close-by IPPs due to the high level of noise and multipath present in the Choke-ring antenna measurements. However, we would still be able to reduce considerably the threat space currently considered in GAST C and D. When using a better quality antenna, MLA, and the Klobuchar model (blue line in Figures 7 and 8), we can detect gradients over $38 \mathrm{~mm} / \mathrm{km}$ using all the data available. The main advantage of this result is that we can use the data obtained from short baselines, i.e. IPPs separated below $10 \mathrm{~km}$. This means that we can measure the value of steep gradients with higher accuracy than in the more conservative scenarios without adding more receivers to the network. Additionally, we lower the value considered for the German threat model approximately 4 times. However, using MLAs would increase enormously the value of the network and may not be necessary if in a particular region we do not need such an accuracy.

\section{CONCLUSIONS AND FUTURE WORK}

In this work we have proposed a real-time ionospheric monitoring approach for single-frequency (L1) single-constellation (GPS) GBAS users, based on a wide area network of multi-frequency multi-constellation GNSS receivers.

First, we explained the functionality of the network. Here, we identified the problem of using measurements containing high levels of noise and multipath and a nominal ionospheric decorrelation for the detection of the gradients with the proposed network.

Then, we evaluated the proposed concept with simulated data. We used realistic values for the noise and multipath from real measurements of a Choke-ring and Multipath Limiting antennas and the nominal ionospheric decorrelation from the Klobuchar model and the $\sigma_{v i g}$ value for Germany. Utilizing this simulated data, we computed the minimum detectable gradient that can be estimated with our network.

Simulation results show that a considerable reduction of the threat space that the remaining monitors, geometry screening in GAST $\mathrm{C}$ and integrity monitoring in GAST D, have to considered seems feasible even while utilizing cheaper antennas for the detection. Furthermore, the main benefit of this approach is that it supports the already existing GAST C and D solutions by reducing their conservatism without changing the overall integrity concept. 
Future work will validate the estimation capability of our concept with data from real gradients and integrate the minimum detectable gradient into the computation of the current GAST C and D integrity parameters. 


\section{References}

[1] RTCA DO-253C. Minimum Operational Performance Standards for GPS Local Area Augmentation System Airborne Equipment. Tech. rep. 253C. Radio Technical Commission for Aeronautics, 2008.

[2] Jiyun Lee et al. "Assessment of Ionosphere Spatial Decorrelation for Global Positioning System-Based Aircraft Landing Systems”. In: Journal of Aircraft 44.5 (2007), pp. 1662-1669. DOI: 10.2514 /1 .28199.

[3] Christoph Mayer et al. "Ionosphere Threat Space Model Assessment for GBAS". In: ION GNSS 2009. 2009. URL: http://elib.dlr.de/60300/.

[4] Jiyun Lee et al. "Position-Domain Geometry Screening to Maximize LAAS Availability in the Presence of Ionosphere Anomalies". In: Proceedings of the ION GNSS. 2006.

[5] Seebany Datta-Barua et al. "Ionospheric Threat Parameterization for Local Area Global-Positioning-System-Based Aircraft Landing Systems”. In: Journal of Aircraft 47.4 (2010), pp. 1141-1151.

[6] ICAO. Standards and Recommended Practices (SARPs) Annex 10. Tech. rep. ICAO, 2017.

[7] RTCA DO-253D. "Minimum Operational Performance Standards for GPS Local Area Augmentation System Airborne Equipment”. In: Tech. rep. 253D. Radio Technical Commission for Aeronautics (2017).

[8] ICAO NSP/3. Proposed amendments to Annex 10: Ground-Based Augmentation System (GBAS) provisions. -WP-3. Montreal, Canada, 2016.

[9] Jiyun Lee et al. "Preliminary Results from Ionospheric Threat Model Development to Support GBAS Operations in the Brazilian Region". In: Proceedings of the 28th International Technical Meeting of the Satellite Division of the Institute of Navigation (ION GNSS+15). 2015.

[10] Moonseok Yoon, Dongwoo Kim, and Jiyun Lee. "Validation of Ionospheric Spatial Decorrelation Observed During Equatorial Plasma Bubble Events”. In: IEEE Transactions on Geoscience and Remote Sensing 55.1 (2017), pp. 261-271.

[11] Moonseok Yoon et al. "Assessment of Equatorial Plasma Bubble Impacts on Ground-Based Augmentation Systems in the Brazilian Region”. In: Proceedings of the 2016 International Technical Meeting of The Institute of Navigation (2016), pp. 368-379.

[12] John A Klobuchar. "Ionospheric time-delay algorithm for single-frequency GPS users". In: IEEE Transactions on aerospace and electronic systems 3 (1987), pp. 325-331.

[13] Pratap Misra and Per Enge. "Global Positioning System: Signals, Measurements and Performance Second Edition”. In: Massachusetts: Ganga-Jamuna Press (2006).

[14] Daniel Gerbeth et al. "Nominal Performance of Future Dual Frequency Dual Constellation GBAS". In: International Journal of Aerospace Engineering 2016 (2016), pp. 1-20. ISSN: 1687-5974. DOI: 10.1155 / 2016/6835282. URL: http://dx.doi.org/10.1155/2016/6835282.

[15] Mihaela-Simona Circiu et al. “Assessment of Different Dual-frequency Dual-constellation GBAS Processing Modes based on Flight Trials". In: Proceedings of ION GNSS+ 2016 (2016).

[16] Mihaela-Simona Circiu et al. "Evaluation of GPS L5 and Galileo E1 and E5a Performance for Future Multifrequency and Multiconstellation GBAS”. In: Navigation 64.1 (2017), pp. 149-163. 